\title{
Monetary integration and inflation preferences: a real options analysis
}

\author{
Frank Strobel* \\ Department of Economics \\ University of Birmingham \\ Birmingham B15 2TT, U.K. \\ (forthcoming, European Economic Review)
}

\begin{abstract}
We use a two-country model where policymakers minimize BarroGordon-type loss functions over inflation, and inflation preferences follow geometric Brownian motions, to characterize and solve the optimal stopping problem describing a given country's decision of whether or not to pursue monetary integration with the other one, and derive the conditions under which monetary integration can, or will never, be an equilibrium outcome in our economy. We then carry out comparative statics analysis on the bounds characterizing these conditions and on the range of relative inflation preference parameters that support monetary integration in equilibrium, and illustrate with numerical examples.

Keywords: monetary integration; inflation preference; real option JEL classification: E5, F3
\end{abstract}

${ }^{*}$ Helpful comments by Harald Uhlig, Gerard Pfann and an anonymous referee are gratefully acknowledged. 


\section{Introduction}

When countries (or regions) evaluate the potential advantages of forming or joining a monetary union and their preferences over inflation differ, conventional wisdom suggests that any one of them will generally benefit from giving up monetary independence if the resulting union's preferences are at least as inflation averse as its own. ${ }^{1}$ This simplistic view is, however, at odds with the growing literature on irreversible investment under uncertainty, which has shown that the decision to invest in an irreversible project with uncertain payoffs can be profoundly affected when that investment can be delayed, as the (real) option of waiting then typically has positive value and needs to be accounted for. ${ }^{2}$

Several papers in the literature have started to apply this real-options methodology to a country's decision of whether or not to proceed with monetary integration (or disintegration) when inflation preferences are stochastic and such a move is interpreted as largely irreversible. ${ }^{3}$ Strobel (2000) uses a simple two-country model where policymakers minimize a Barro-Gordontype loss function over inflation to examine the value of the option of monetary integration when the national preference parameters associated with an inflationary surprise follow geometric Brownian motions. Deriving analytically the critical level of the ratio of these parameters that triggers a move to monetary integration, it finds that a country will be willing to give up monetary independence only if the other country is valuing inflationary surprises strictly, and potentially substantially, less than itself. The main drawbacks of that paper are the strictly partial nature of its framework, which critically implies that monetary integration is never actually an equilibrium outcome there, and the fact that countries' weights in the determination of the union-wide inflation preference are restricted to a symmetric scenario, which

\footnotetext{
${ }^{1}$ This abstracts from the other potential costs and benefits of monetary integration; see e.g. De Grauwe (2000), Gros/Thygesen (1998).

${ }^{2}$ See e.g. Dixit (1992), Pindyck (1991) or, more comprehensively, Dixit/Pindyck (1994).

${ }^{3}$ The growing political economy literature on the break-up of nations (see e.g. Bolton/Roland/Spolaore (1996), Bolton/Roland (1997) or Fidrmuc (1999)) focusses on related issues without, so far, taking the real-options nature of a secession decision into account when its payoffs are uncertain and a degree of irreversibility applies.
} 
severely limits its practical applicability. Similar (partial) frameworks are also used in Strobel (2001), which examines the value of the option of monetary disintegration for a country in an existing monetary union when the national inflation preference parameters follow a similar stochastic process, and Strobel (2002), which extends the previous paper by deriving explicit closed-form solutions for the expected time and probability for a country in an existing monetary union to want to return to monetary independence.

Our present paper builds on and extends Strobel (2000) and in the process addresses the two major shortcomings prevailing in the existing literature. Firstly, by taking equilibrium considerations into account explicitly, we are able to characterize the conditions determining whether monetary integration can, or will never, be an equilibrium outcome in our economy. Secondly, by allowing for countries' (or regions') weights in the determination of the union-wide inflation preference to be of different magnitudes, we are also able to, e.g., capture the relevant scenario of a single country joining a pre-existing monetary union made up of several other countries. The paper thus proceeds as follows: we begin by illustrating and motivating its main ideas and intuition with an example in Section 2. Section 3 then outlines a two-country model where national and supranational policymakers minimize a Barro-Gordon-type loss function over inflation, with inflation preferences modelled as following geometric Brownian motions, ${ }^{4}$ and solve for the loss functions applying in the monetary independence and integration cases, respectively, that result from policymakers' optimal setting of inflation in a discretionary policy scenario under rational expectations. In Section 4 we characterize and solve the optimal stopping problem that describes a given country's decision of whether or not to pursue monetary integration with the other one, and derive the conditions under which monetary integration can, or will never, be an equilibrium outcome in our economy; these are seen to be crucially influenced by the relative importance of the value of the option of monetary integration versus the magnitude of the other (net)

\footnotetext{
${ }^{4}$ The empirical literature appears still without consensus over whether a country's inflation is better described by a non-stationary or a stationary process, see e.g. Culver/Papell (1997), Lai (1997); a geometric Brownian motion for the inflation process, unlike e.g. a mean-reverting one, allows for closed-form solutions, and is thus used for analytical ease.
} 
benefits associated with such a move. Comparative statics analysis on the bounds characterizing these conditions, and on the range of relative inflation preference parameters that support monetary integration in equilibrium, is consequently carried out in Section 5. We find that for both countries to agree on forming a monetary union, and thus for monetary integration to be an equilibrium outcome, their respective inflation preferences need to be more similar the more variable and the less correlated these are, and also the lower are the discount rate and the other (net) benefits associated with such a move. Section 6 illustrates the analytical results obtained in the two preceding sections with some numerical examples; it also shows the range of relative inflation preferences supporting monetary integration in equilibrium to be (roughly) decreasing in the degree of similarity of the two countries' (or regions') weights in the determination of the union-wide inflation preference. Section 7 then sums up and concludes the paper.

\section{Intuition and example}

Consider the following scenario: the United Kingdom, say, is not yet a member of the European Monetary Union, but is evaluating whether to join or not; analogously, the current EMU countries are considering whether or not to admit the U.K. into the union. Policymakers are concerned with inflation performance as a time inconsistency problem in monetary policymaking causes an undesirable inflation bias to persist. If the U.K.'s inflation preferences were lower than the EMU area's, it would benefit from giving up monetary independence only if other (net) benefits of monetary integration compensated for the resulting worsening in inflation performance (a result of some bargaining process); the EMU countries, on the other hand, would benefit unequivocally as their inflation performance would improve in this case. Now, if the future evolution of these inflation preferences were uncertain, it may be beneficial to postpone forming a monetary union as both parties will be more reluctant to commit to an irreversible move that might later prove less advantageous than initially thought. This value of waiting, arising from the real options nature of the decision problem involved and 
well-known from the literature on irreversible investment under uncertainty, can be substantial; it needs to be properly accounted for as it can significantly affect the conditions under which, in our example, both the U.K. and the EMU countries would find it beneficial to go ahead with monetary integration. Generally, the more uncertain those inflation preferences are and the more asynchronously they evolve, i.e. the more variable and asymmetric are the underlying economic and political shocks driving them, the more similar they then need to be for the U.K. and the EMU countries to mutually agree on forming a monetary union. The two sides' inflation preferences can be allowed to be more dissimilar, on the other hand, the more myopic policymakers are, as a presently beneficial move towards monetary integration turning less advantageous in the future will matter less to them in that case, and also the higher are the other (net) benefits resulting from a move towards a monetary union. As the U.K.'s contribution in the determination of the union-wide inflation preference, as a result of the likely decision making process, will be relatively small, it will either require the EMU countries to have a significantly lower inflation preference than its own or, as in our above scenario, require significant compensation in terms of the other (net) benefits of monetary integration to make up for the resulting worsening in inflation performance. For a given level of those other (net) benefits, the EMU countries, on the other hand, would be willing to tolerate a possibly significantly higher inflation preference on the U.K.'s part as the corresponding inflation preference in the wider union will be dominated and largely determined by their own one. These qualitative predictions are made analytically concrete in the following sections that, in a two-country model, characterize and solve the optimal stopping problems describing countries' (or regions') decisions of whether or not to pursue monetary integration with each other when inflation preferences are stochastic and such a move is interpreted as largely irreversible. 


\section{Inflation preferences and loss functions}

The national and supranational policymakers' objectives, for countries (or regions) $a, b$ and the union $u$, are taken to involve the instantaneous loss rate

$$
l\left(\beta_{i}, \pi_{i}, t\right)=\left[\pi_{i}(t)\right]^{2}-\beta_{i}(t)\left[\pi_{i}(t)-\pi_{i}^{e}(t)\right] \quad, \quad i=a, b, u
$$

where $\pi_{i}(t)$ and $\pi_{i}^{e}(t)$ represent inflation and expected inflation, respectively. ${ }^{5}$

For the case of monetary independence the national policymakers' inflation preference (or benefit) parameters $\beta_{j}(t) \geq 0, j=a, b$ are assumed to follow geometric Brownian motions without drift ${ }^{6}$

$$
d \beta_{j}=\sigma \beta_{j} d z_{j}
$$

where $\sigma \geq 0,{ }^{7} d z_{j}=\varepsilon_{j}(t) \sqrt{d t}$ are increments of Wiener processes with $\varepsilon_{j}(t) \sim \operatorname{NID}(0,1)$, and $E_{t}\left(d z_{a} d z_{b}\right)=\rho d t$ with $\rho$ the coefficient of correlation between the processes $z_{j}$ (thus $-1 \leq \rho \leq 1$ ).

For the monetary integration case the supranational policymaker's inflation preference parameter is likely to be the outcome of a bargaining process: we assume that it is determined as $\beta_{u}(t)=\left[\beta_{a}(t)\right]^{\omega}\left[\beta_{b}(t)\right]^{1-\omega}$, with $0<\omega<1$, a weighted geometric average of the constituent national inflation preference parameters $\beta_{j}(t)$ evolving as above. ${ }^{8}$ The weights $\omega$ could e.g. be thought of as reflecting relative size, bargaining power, or number of seats on a decision-making committee; this model thus also captures the scenario of a single country joining a pre-existing monetary union made up of several other countries. Note that our particular specification implies a disinflationary bias in the supranational policymaker's inflation preference, from

Lemma 1 The instantaneous drift rate of $\beta_{u}(t)$ is strictly negative for $\sigma>$

\footnotetext{
${ }^{5}$ This adapts the discrete-time setup in Barro/Gordon (1983) to a continuous-time environment; similar frameworks are used in Strobel (2000, 2001, 2002).

${ }^{6}$ See footnote 4 .

${ }^{7}$ The common instantaneous variance rate of $\beta_{j}(t)$ is $\sigma^{2}$; in Strobel (2000), variance rates are country-specific.

${ }^{8}$ In Strobel (2000), these weights are restricted to be symmetric.
} 
$0, \rho<1$.

Proof. Using Ito's Lemma, we obtain $d \beta_{u}=\left(-\sigma^{2}(1-\rho) \omega(1-\omega) d t+\right.$ $\left.\sigma\left(\omega d z_{a}+(1-\omega) d z_{b}\right)\right) \beta_{u}$; thus $\frac{E\left[d \beta_{u}\right]}{\beta_{u}}=-\sigma^{2}(1-\rho) \omega(1-\omega) d t<0$ for $\sigma>0, \rho<1$.

Restricting our analysis to a discretionary policy scenario under rational expectations, ${ }^{9}$ the policymakers' choice problems are to solve for the optimal feedback rule $\pi_{i}^{*}\left(\beta_{i}\right)$ that satisfies the loss function

$$
L\left(\beta_{i}, t\right)=\min _{\pi_{i}} E_{t} \int_{t}^{\infty} l\left(\beta_{i}, \pi_{i}, \tau\right) e^{-\mu(\tau-t)} d \tau \quad, \quad i=a, b, u
$$

where $\mu>0$ is the common discount rate, treating inflationary expectations $\pi_{i}^{e}(\tau)$ as given $\forall \tau \geq t$. We then obtain

Lemma 2 In a rational expectations equilibrium, the loss functions associated with the national and supranational policymakers are

$$
\begin{aligned}
L\left(\beta_{j}, t\right) & =\frac{1}{4\left(\mu-\sigma^{2}\right)}\left[\beta_{j}(t)\right]^{2} \quad, \quad j=a, b \\
L\left(\beta_{u}, t\right) & =\frac{1}{4\left(\mu-\sigma^{2}(1-4(1-\rho) \omega(1-\omega))\right)}\left[\beta_{a}(t)\right]^{2 \omega}\left[\beta_{b}(t)\right]^{2(1-\omega)}
\end{aligned}
$$

respectively, for $\mu-\sigma^{2}>0$.

Proof. Using the relevant Bellman equation $\mu L\left(\beta_{i}, t\right)=\min _{\pi_{i}}\left[l\left(\beta_{i}, \pi_{i}, t\right)+\right.$ $\left.\frac{1}{d t} E_{t} d L\left(\beta_{i}, t\right)\right]$ and applying Ito's Lemma, we obtain $\pi_{i}^{*}\left(\beta_{i}\right)=\frac{\beta_{i}(t)}{2}$ as the optimal feedback rule in question. Imposing rational expectations such that $\pi_{i}^{e}(\tau)=\pi_{i}(\tau), \forall \tau \geq t$ at this stage and using standard properties ${ }^{10}$ of geometric Brownian motion to simplify, the loss functions (4) and (5) hold in equilibrium, as long as $\mu-\sigma^{2}>0$ for convergence.

These loss functions thus give policymakers' expected present discounted value of losses associated with current and future inflation when those are set optimally in a discretionary policy scenario under rational expectations.

\footnotetext{
${ }^{9}$ We abstract from the role of reputation in the repeated policy game and focus on a basic scenario where an inflation bias does exist in equilibrium.

${ }^{10}$ See e.g. Dixit (1993, eq. (2.7)).
} 


\section{Optimal stopping problem and solution}

Starting from a situation of monetary independence, the decision of, say, country $a$ on whether or not to pursue monetary integration with country $b$ involves solving the Bellman equation for the optimal stopping problem

$$
F\left(L_{a}, L_{u}\right)=\max \left\{(1+\tau) L_{a}-L_{u}, \frac{1}{\mu d t} E_{t}\left[d F\left(L_{a}, L_{u}\right)\right]\right\}
$$

where $F\left(L_{a}, L_{u}\right)$ is the value to country $a$ of the option of monetary integration with country $b$, the proportionality factor $\tau \geq 0$ encompasses all other (net) benefits of monetary integration, ${ }^{11}$ and $(1+\tau) L_{a}-L_{u}$ is the expected discounted benefit of entering into such an arrangement. ${ }^{12}$ Analogously, country $b$ evaluates a similar optimal stopping problem to decide whether or not to pursue monetary integration with country a. Monetary integration is then an equilibrium outcome in this economy only if both countries $a$ and $b$ are willing to exercise their respective options together at a given point in time, while monetary independence remains the status quo if at least one of the countries prefers to leave the option of monetary integration unexercised for the time being. Note that this formulation implicitly assumes that monetary integration is an irreversible process, so that the costs of a later return to monetary independence are deemed prohibitively high. ${ }^{13} \mathrm{We}$ can then obtain

Proposition 3 Monetary integration is an equilibrium outcome in this economy iff

$$
\Upsilon_{a} \leq \frac{\beta_{a}}{\beta_{b}} \leq \frac{1}{\Upsilon_{b}}
$$

with trigger levels ${ }^{14}$

$$
\Upsilon_{a}=\left(\Xi_{a}(1+\tau)^{-1}\right)^{\frac{1}{2(1-\omega)}}, \Upsilon_{b}=\left(\Xi_{b}(1+\tau)^{-1}\right)^{\frac{1}{2 \omega}}
$$

\footnotetext{
${ }^{11}$ See e.g. De Grauwe (2000), Gros/Thygesen (1998); these are ignored in Strobel (2000).

${ }^{12}$ We use $L_{i} \equiv L\left(\beta_{i}, t\right)$ for ease of notation.

${ }^{13}$ The abandonment option of possible future monetary disintegration could be included, albeit at considerable analytical cost.

${ }^{14}$ Note that, for $\tau=0 \wedge \omega=\frac{1}{2}$, the trigger values $\Upsilon_{a}, \Upsilon_{b}$ coincide with the special case of eq. (10) in Strobel (2000).
} 
where

$$
\begin{aligned}
& \Xi_{a} \equiv \frac{\mu-\sigma^{2}}{\mu+\sigma\left(\sigma(1-2(\rho+(1-\rho) \omega))-2 \sqrt{(1-\rho)\left(\mu-\rho \sigma^{2}\right)}(1-\omega)\right)} \\
& \Xi_{b} \equiv \frac{\mu-\sigma^{2}}{\mu+\sigma\left(\sigma(1-2(\rho+(1-\rho)(1-\omega)))-2 \sqrt{(1-\rho)\left(\mu-\rho \sigma^{2}\right)} \omega\right)}
\end{aligned}
$$

For $\sigma>0 \wedge \rho<1$, it holds that $\Xi_{a}>1, \Xi_{b}>1 ; \Xi_{a}=1, \Xi_{b}=1$ apply for $\sigma=0 \vee \rho=1$.

Proof. For country $a$, postponing monetary integration for a further instant $d t$ is optimal in the continuation region of the optimal stopping problem eq. (6), giving the relevant Bellman equation as

$$
\mu F\left(L_{a}, L_{u}\right)=\frac{1}{d t} E_{t}\left[d F\left(L_{a}, L_{u}\right)\right]
$$

Applying Ito's Lemma to eq. (7) and noting that the value function $F\left(L_{a}, L_{u}\right)$ should be homogeneous of degree $1,{ }^{15}$ so that $F\left(L_{a}, L_{u}\right)=L_{u} f(\Gamma)$ where $\Gamma \equiv \frac{L_{a}}{L_{u}},{ }^{16}$ we obtain

$$
\begin{aligned}
4 \sigma^{2}(1-\rho)(1-\omega)^{2} \Gamma^{2} \frac{\partial^{2} f}{\partial \Gamma^{2}}+ & 4 \sigma^{2}(1-\rho)(1-\omega) \omega \Gamma \frac{\partial f}{\partial \Gamma} \\
& -\left(\mu-\sigma^{2}(1-4(1-\rho)(1-\omega) \omega)\right) f=0
\end{aligned}
$$

as the differential equation that characterizes the evolution of $f(\Gamma)$ in that region.

We solve equation (8) by standard methods, using the value-matching and smooth-pasting conditions $f\left(\Gamma^{*}\right)=(1+\tau) \Gamma^{*}-1$ and $\frac{\partial f\left(\Gamma^{*}\right)}{\partial \Gamma}=(1+\tau)$,

\footnotetext{
${ }^{15}$ This adopts the solution strategy in Dixit/Pindyck (1994, p. 210).

${ }^{16}$ Thus, $\Gamma=\frac{\mu-\sigma^{2}(1-4(1-\rho) \omega(1-\omega))}{\mu-\sigma^{2}}\left(\frac{\beta_{a}}{\beta_{b}}\right)^{2(1-\omega)}$ from eq. (4) and (5).
} 
plus the boundary condition $f(0)=0,{ }^{17}$ and thus obtain

$$
\begin{aligned}
\Gamma^{*} & =\frac{\beta_{1}}{(1+\tau)\left(\beta_{1}-1\right)} \\
\text { where } \beta_{1} & =\frac{(1-\rho) \sigma(1-2 \omega) \sqrt{\Theta}+\sqrt{(1-\rho)\left(\mu-\rho \sigma^{2}\right) \Theta}}{2(1-\rho) \sigma(1-\omega) \sqrt{\Theta}}>1 \\
\text { with } \Theta & \equiv \mu-\sigma^{2}(1-4(1-\rho)(1-\omega) \omega)
\end{aligned}
$$

as the critical (trigger) value $\Gamma^{*}$. From the definition of $\Gamma$ it then follows that $\Upsilon_{a}=\left(\frac{\left(\mu-\sigma^{2}\right)(1+\tau)^{-1}}{\left(\mu+\sigma\left(\sigma(1-2(\rho+(1-\rho) \omega))-2 \sqrt{(1-\rho)\left(\mu-\rho \sigma^{2}\right)}(1-\omega)\right)\right)}\right)^{\frac{1}{2(1-\omega)}}$ is the trigger value of relative inflation preference parameters $\frac{\beta_{a}}{\beta_{b}}$ separating the region in $\left(\beta_{a}, \beta_{b}\right)$ space where country $a$ 's option of monetary integration remains unexercised (i.e. for $\frac{\beta_{a}}{\beta_{b}}<\Upsilon_{a}$ ) from the one where exercise of that option is perceived as desirable (i.e. for $\frac{\beta_{a}}{\beta_{b}} \geq \Upsilon_{a}$ ).

Analogously, repeating the above solution strategy for country $b$, we can obtain $\Upsilon_{b}=\left(\frac{\left(\mu-\sigma^{2}\right)(1+\tau)^{-1}}{\left(\mu+\sigma\left(\sigma(1-2(\rho+(1-\rho)(1-\omega)))-2 \sqrt{(1-\rho)\left(\mu-\rho \sigma^{2}\right)} \omega\right)\right)}\right)^{\frac{1}{2 \omega}}$ as the trigger value of relative inflation preference parameters $\frac{\beta_{b}}{\beta_{a}}$ determining whether country $b$ 's option of monetary integration remains unexercised (i.e. for $\frac{\beta_{b}}{\beta_{a}}<\Upsilon_{b}$ ) or whether exercise of that option is perceived as desirable (i.e. for $\frac{\beta_{b}}{\beta_{a}} \geq \Upsilon_{b}$ ).

Monetary integration will then be an equilibrium outcome in this economy only if both countries $a$ and $b$ are willing to exercise their respective options concurrently, requiring

$$
\Upsilon_{a} \leq \frac{\beta_{a}}{\beta_{b}} \leq \frac{1}{\Upsilon_{b}}
$$

to hold.

It can finally be shown that $\Xi_{a}>1, \Xi_{b}>1$ for $\sigma>0 \wedge \rho<1$ and the assumptions on $\omega, \tau, \mu$ from above, while $\Xi_{a}=1, \Xi_{b}=1$ follow by substitution for $\sigma=0 \vee \rho=1$.

Country a perceives exercise of the option of monetary integration as desirable only when the current value of relative inflation preference parameters

\footnotetext{
${ }^{17}$ Note that zero is an absorbing barrier for the geometric Brownian motion $\Gamma$.
} 
$\frac{\beta_{a}}{\beta_{b}}$ is greater than (or equal to) its trigger value $\Upsilon_{a}$; intuitively, the higher a country's inflation preference relative to the other's, the more it stands to gain from giving up its monetary independence. For the case where $\frac{\beta_{a}}{\beta_{b}}<\Upsilon_{a}$ , on the other hand, country $a$ strictly prefers to leave the option of monetary integration unexercised for the time being. Analogously, country $b$ views exercise of the option of monetary integration as desirable only when $\frac{\beta_{b}}{\beta_{a}} \geq \Upsilon_{b}$, while it strictly prefers the status quo of monetary independence for the case where $\frac{\beta_{b}}{\beta_{a}}<\Upsilon_{b}$. Both countries $a$ and $b$ need to be willing to exercise their respective options at the same point in time for monetary integration to be an equilibrium outcome in this economy; thus, the current value of relative inflation preference parameters $\frac{\beta_{a}}{\beta_{b}}$ needs to be within the bounds given by Proposition 3 for this to happen. The trigger values $\Upsilon_{a}, \Upsilon_{b}$ determining these bounds are seen to be crucially influenced by the relative importance of the value of the option of monetary integration (as embodied in $\Xi_{a}, \Xi_{b}$ ) versus the magnitude of the other (net) benefits associated with such a move (as represented by $\tau$ ). We can state

Corollary 4 If $\Xi_{j} \leq 1+\tau$ for $j=a, b$ (as e.g. for $\sigma=0$ or $\rho=1$ ), it holds that $\Upsilon_{j} \leq 1$; monetary integration can be an equilibrium outcome in this case.

Proof. The inequality for $\Upsilon_{j}$ follows straightforwardly given the assumptions on $\omega$.

When the value of the option of monetary integration is sufficiently small compared to the other (net) benefits of such a move, the trigger value $\Upsilon_{j}$ is less than (or equal to) one; thus, a country may be willing to pursue monetary integration even if the other country's inflation preferences are higher than its own. If (but not only if) this holds for both countries, monetary integration can be an equilibrium outcome in this case. This applies in particular for the "traditional" scenarios where there is no uncertainty (i.e. $\sigma=0$ ) or the two countries' inflation preferences are perfectly correlated (i.e. $\rho=1$ ); the value of the option of monetary integration is zero in both cases (implying $\Xi_{a}=1, \Xi_{b}=1$ ), leaving the other (net) benefits of such a move as the sole determinant of the trigger values $\Upsilon_{j}$. We can further state 
Corollary 5 If $\Xi_{j}>1+\tau$ for $j=a, b$ (as e.g. for $\sigma>0, \rho<1$ and $\tau=0$ ), it holds that $\Upsilon_{j}>1$; monetary integration can never be an equilibrium outcome in this case.

Proof. This follows analogously to Corollary 4.

When the other (net) benefits associated with monetary integration are sufficiently small compared to the value of the option of monetary integration, the trigger value $\Upsilon_{j}$ is greater than one; thus, a country will be willing to pursue monetary integration only if the other country's inflation preferences are lower than its own. If (but not only if) this holds for both countries, monetary integration can never be an equilibrium outcome in this case. This applies e.g. for the scenario where there is some uncertainty (i.e. $\sigma>0$ ), the two countries' inflation preferences are less than perfectly correlated (i.e. $\rho<1$ ) but the other (net) benefits of monetary integration are zero; ${ }^{18}$ the value of the option of monetary integration is positive in this case (implying $\left.\Xi_{a}>1, \Xi_{b}>1\right)$ and constitutes the sole determinant of the trigger values $\Upsilon_{j}$

\section{Comparative statics}

Turning now to a comparative statics analysis of the trigger levels $\Upsilon_{a}$ and $\Upsilon_{b}$, for the non-degenerate case where $\sigma>0$ and $\rho<1$, we obtain

Proposition 6 The directional impact of changes in $\sigma, \rho, \mu, \tau$ and $\omega$ on

\footnotetext{
${ }^{18}$ This coincides with the framework used in Strobel (2000); thus, monetary integration can never be an equilibrium outcome there.
} 
the trigger levels $\Upsilon_{a}$ and $\Upsilon_{b}$ is

$$
\begin{aligned}
& \frac{\partial \Upsilon_{a}}{\partial \sigma}>0, \frac{\partial \Upsilon_{b}}{\partial \sigma}>0 \\
& \frac{\partial \Upsilon_{a}}{\partial \rho}<0, \frac{\partial \Upsilon_{b}}{\partial \rho}<0 \\
& \frac{\partial \Upsilon_{a}}{\partial \mu}<0, \frac{\partial \Upsilon_{b}}{\partial \mu}<0 \\
& \frac{\partial \Upsilon_{a}}{\partial \tau}<0, \frac{\partial \Upsilon_{b}}{\partial \tau}<0 \\
& \frac{\partial \Upsilon_{a}}{\partial \omega}<0, \frac{\partial \Upsilon_{b}}{\partial \omega}>0
\end{aligned}
$$

for $\sigma>0 \wedge \rho<1$.

Proof. We can show that $\frac{\partial \Upsilon_{a}}{\partial \sigma}=-\frac{\mu(1-\rho) \Psi(1+\tau)\left(\frac{\Xi_{a}}{1+\tau}\right)^{1+\frac{1}{2-2 \omega}}}{\left(\mu-\sigma^{2}\right)^{2} \sqrt{(1-\rho)\left(\mu-\rho \sigma^{2}\right)}}>0$, with $\Xi_{a}>0$ and $\Psi \equiv-\mu+\sigma\left((2 \rho-1) \sigma+2 \sqrt{(1-\rho)\left(\mu-\rho \sigma^{2}\right)}\right)<0$ for $\sigma>0$ , $\rho<1$ and the assumptions on $\omega, \tau, \mu$ from above.

It can be similarly seen that $\frac{\partial \Upsilon_{a}}{\partial \rho}=\frac{\sigma \Psi(1+\tau)\left(\frac{\Xi_{a}}{1+\tau}\right)^{1+\frac{1}{2-2 \omega}}}{2\left(\mu-\sigma^{2}\right) \sqrt{(1-\rho)\left(\mu-\rho \sigma^{2}\right)}}<0$ and $\frac{\partial \Upsilon_{a}}{\partial \mu}=$ $\frac{(1-\rho) \sigma \Psi(1+\tau)\left(\frac{\Xi_{a}}{1+\tau}\right)^{1+\frac{1}{2-2 \omega}}}{2\left(\mu-\sigma^{2}\right)^{2} \sqrt{(1-\rho)\left(\mu-\rho \sigma^{2}\right)}}<0$.

Also, we can show that $\frac{\partial \Upsilon_{a}}{\partial \tau}=-\frac{\Upsilon_{a}}{2(1+\tau)(1-\omega)}<0$.

Further, we can demonstrate that $\frac{\partial \Upsilon_{a}}{\partial \omega}=\frac{2\left(\frac{\Xi_{a}}{1+\tau}\right)^{\frac{1}{2-2 \omega}} \Phi}{(2-2 \omega)^{2}}<0$, where $\Phi \equiv$ $\frac{2(1-\rho) \sigma(1-\omega)}{-\sqrt{(1-\rho)\left(\mu-\rho \sigma^{2}\right)}+(1-\rho) \sigma(1-2 \omega)}+\log \left(\frac{\Xi_{a}}{1+\tau}\right) ;$ using $\log (x)<x-1$ for $x>0 \wedge x \neq 1$ ${ }^{19} \Phi<\frac{-\tau \Xi_{a}}{1+\tau} \leq 0$ for $\Xi_{a} \neq 1+\tau$, and $\Phi=\frac{-\tau \Xi_{a}}{1+\tau}<0$ for $\Xi_{a}=1+\tau$.

With $\Upsilon_{b}=\left.\Upsilon_{a}\right|_{\omega \rightarrow 1-\omega}$, replacing $\omega$ with $1-\omega$ throughout, $\frac{\partial \Upsilon_{b}}{\partial \mu}=$ $\left.\frac{\partial \Upsilon_{a}}{\partial \mu}\right|_{\omega \rightarrow 1-\omega}<0, \frac{\partial \Upsilon_{b}}{\partial \sigma}=\left.\frac{\partial \Upsilon_{a}}{\partial \sigma}\right|_{\omega \rightarrow 1-\omega}>0, \frac{\partial \Upsilon_{b}}{\partial \tau}=\left.\frac{\partial \Upsilon_{a}}{\partial \tau}\right|_{\omega \rightarrow 1-\omega}<0, \frac{\partial \Upsilon_{b}}{\partial \rho}=$ $\left.\frac{\partial \Upsilon_{a}}{\partial \rho}\right|_{\omega \rightarrow 1-\omega}<0$ and $\frac{\partial \Upsilon_{b}}{\partial \omega}=-\left.\frac{\partial \Upsilon_{a}}{\partial \omega}\right|_{\omega \rightarrow 1-\omega}>0$ then hold.

We observe that $\Upsilon_{a}, \Upsilon_{b}$ are increasing in the variance rate $\sigma$, a result that is familiar from the standard (financial) option pricing literature; higher uncertainty regarding country $a$ and $b$ 's inflation preferences increases the value of the option of monetary integration and thereby raises the trigger value that prompts that option to be exercised. The trigger values $\Upsilon_{a}, \Upsilon_{b}$

\footnotetext{
${ }^{19} \mathrm{My}$ thanks to Ralph Bailey for pointing this out.
} 
are decreasing in the correlation coefficient $\rho$, as the likelihood of the two countries' inflation preference parameters drifting apart gets smaller the more correlated these are; this decreases the value of the option to pursue monetary integration. Increasing the discount rate $\mu$ also leads to lower levels of $\Upsilon_{a}, \Upsilon_{b}$ : a higher discount rate (i.e. policymakers being more short-sighted) raises the opportunity cost of leaving the option of monetary integration unexercised for a further instant, and thus decreases the value of that option. ${ }^{20}$ The trigger values $\Upsilon_{a}, \Upsilon_{b}$ are further decreasing in the proportionality factor $\tau$, as exercising the option of monetary integration becomes more rewarding the higher the other (net) benefits associated with such a move. Lastly, the higher a country's weight in the determination of the union-wide inflation preference, e.g. the higher $\omega$ for country $a$, the more similar are the inflation performance under monetary integration and independence scenarios for that country; this reduces the value of the option of monetary integration and thus lowers the trigger value prompting its exercise.

Further extending our comparative statics analysis to the range of relative inflation preference parameters $\frac{\beta_{a}}{\beta_{b}}$ that support a move towards monetary integration in equilibrium, we can state

Corollary 7 The directional impact of changes in $\sigma, \rho, \mu$ and $\tau$ on the range of relative inflation preference parameters $\frac{\beta_{a}}{\beta_{b}}$ that support monetary integration as an equilibrium outcome is

$$
\begin{aligned}
& \frac{\partial\left(\frac{1}{\Upsilon_{b}}-\Upsilon_{a}\right)}{\partial \sigma}<0 \\
& \frac{\partial\left(\frac{1}{\Upsilon_{b}}-\Upsilon_{a}\right)}{\partial \rho}>0 \\
& \frac{\partial\left(\frac{1}{\Upsilon_{b}}-\Upsilon_{a}\right)}{\partial \mu}>0 \\
& \frac{\partial\left(\frac{1}{\Upsilon_{b}}-\Upsilon_{a}\right)}{\partial \tau}>0
\end{aligned}
$$

\footnotetext{
${ }^{20}$ These results are qualitatively similar to the ones obtained in Strobel (2000).
} 
for $\sigma>0 \wedge \rho<1$.

Proof. This follows straightforwardly from Proposition 6.

We note that higher uncertainty regarding country $a$ and $b$ 's inflation preferences narrows the range of relative inflation preference parameters $\frac{\beta_{a}}{\beta_{b}}$ that give rise to monetary integration in equilibrium; thus, for the two countries to agree on moving towards a monetary union, their respective inflation preferences need to be more similar the more uncertainty is attached to their future evolution. The range of country $a$ and $b$ 's relative inflation preferences that is compatible with monetary integration being an equilibrium outcome is, on the other hand, larger the more correlated these are; the more "in-sync" those inflation preferences evolve, the more dissimilar they can be for the two countries to still agree on going ahead with monetary integration. The higher the discount rate (i.e. the more short-sighted policymakers), the less important is the uncertainty surrounding the future evolution of our countries' inflation preferences compared to the potential instantaneous benefits from a move towards monetary integration, and thus the less similar those inflation preferences need to be for that move to be mutually beneficial. Also, the larger the other (net) benefits associated with monetary integration, the less the relative importance of the (real) option value associated with such a move, and thus the higher the degree of dissimilarity of country $a$ and $b$ 's inflation preferences that is still compatible with monetary integration occurring in equilibrium. Finally, the comparative statics effects of changes in a country's weight in the determination of the union-wide inflation preference on the relative range of (national) inflation preferences that are consistent with monetary integration in equilibrium are not easily analytically tractable, and will be discussed on the basis of some numerical examples in the next section.

\section{$6 \quad$ Numerical examples}

To illustrate the analytical results obtained in Sections 4 and 5 further, we graph the trigger values $\Upsilon_{a}$ (solid line) and $\frac{1}{\Upsilon_{b}}$ (dashed line) for different 
parameter combinations of $\sigma, \rho, \mu, \tau$ and $\omega$ in Figures 1-4.

We observe throughout Figures 1-4 that for certain parameter combinations $\Upsilon_{a}>\frac{1}{\Upsilon_{b}}$ applies; monetary integration can never be an equilibrium outcome in these circumstances as countries $a$ and $b$ will never be willing to exercise their respective options of monetary integration at the same time. For other parameter combinations, however, we note that $\Upsilon_{a} \leq \frac{1}{\Upsilon_{b}}$ holds; monetary integration can generally be an equilibrium outcome in these cases and countries will want to exercise their respective options of monetary integration concurrently if their relative inflation preferences fall within the exercise range given by Proposition 3.

Figure 1 illustrates how higher uncertainty regarding country $a$ and $b$ 's inflation preferences increases the respective trigger levels $\Upsilon_{a}, \Upsilon_{b}$ and thereby narrows the range of relative inflation preference parameters $\frac{\beta_{a}}{\beta_{b}}$ that are consistent with monetary integration as an equilibrium outcome; for sufficiently high levels of uncertainty, monetary integration will never arise in equilibrium. Figure 2 demonstrates that the trigger levels $\Upsilon_{a}, \Upsilon_{b}$ are decreasing in the degree of correlation between the two countries' inflation preferences, thus leading to a widening of the range of relative inflation preference parameters that support a move towards monetary integration in equilibrium; the less correlated the two countries' inflation preferences, the less likely it is that monetary integration can ever occur in equilibrium. In figure 3 , the trigger levels $\Upsilon_{a}, \Upsilon_{b}$ are seen to be also decreasing in the discount rate, again extending the range of relative inflation preference parameters that prompt monetary integration as an equilibrium outcome; the lower the discount rate, the smaller the set of scenarios where monetary integration can ever arise in equilibrium. Similarly, an increase in the other (net) benefits associated with monetary integration is seen to lower the trigger levels $\Upsilon_{a}, \Upsilon_{b}$ in Figure 4, thereby enlarging the range of relative inflation preference parameters that give rise to such a move in equilibrium; the lower the level of these other (net) benefits, the more likely it is that monetary integration is never supported in equilibrium.

Finally, an increase in a country's weight in the determination of the union-wide inflation preference can be seen in Figures 1-4 to reduce that 
country's trigger level $\Upsilon_{j}$, and increase the other country's correspondingly. The range of relative inflation preference parameters that support monetary integration in equilibrium is here shown to be (roughly) decreasing in the degree of similarity of the two countries' weights in the determination of the union-wide inflation preference. ${ }^{21}$ This last result can be explained by the fact that the more dominant a country becomes in the determination of the union-wide inflation preference, the more similar the inflation performance under monetary integration and independence scenarios are for that country, and thus the more tolerant it becomes towards potentially higher inflation preferences in the other one; monetary integration is therefore more likely to occur in equilibrium the more dissimilar are countries' (or regions') weights in the determination of the union-wide inflation preference.

\section{Conclusion}

Our paper applied real-options methodology to countries' (or regions') decisions of whether or not to form (or join) a monetary union when their inflation preferences are stochastic and such a move is considered as largely irreversible. Building on and extending previous work by Strobel (2000), we used a two-country model where national and supranational policymakers minimize Barro-Gordon-type loss functions over inflation, with inflation preferences modelled as following geometric Brownian motions, and solved for the respective loss functions applying in the monetary independence and integration cases that result from policymakers' optimal setting of inflation in a discretionary policy scenario under rational expectations. We then characterized and solved the optimal stopping problem that describes a given country's decision of whether or not to pursue monetary integration with the other one, and derived the conditions under which monetary integration can, or will never, be an equilibrium outcome in our economy; these were seen to be crucially influenced by the relative importance of the value of the (real) option of monetary integration versus the magnitude of the other

\footnotetext{
${ }^{21}$ Numerical experimentation shows that the minimum of $\frac{1}{\Upsilon_{b}}-\Upsilon_{a}$ generally occurs close to, but rarely precisely for, $\omega=\frac{1}{2}$.
} 
(net) benefits associated with such a move. Comparative statics analysis on the bounds characterizing these conditions, and on the range of relative inflation preference parameters that support monetary integration in equilibrium, was consequently carried out. We found that for both countries to agree on forming a monetary union, and thus for monetary integration to be an equilibrium outcome in our economy, their respective inflation preferences need to be more similar the more variable and the less correlated these are, and also the lower are the discount rate and the other (net) benefits associated with such a move. We finally illustrated the analytical results obtained with some numerical examples; these also showed the range of relative inflation preferences that support monetary integration in equilibrium to be (roughly) decreasing in the degree of similarity of the two countries' (or regions') weights in the determination of the union-wide inflation preference.

\section{References}

[1] Barro, R.J. and D.B. Gordon, 1983, "Rules, discretion and reputation in a model of monetary policy", Journal of Monetary Economics, 12, p. 101-121.

[2] Bolton, P., Roland, G. and E. Spolaore, 1996, "Economic Theories of the Break-Up and Integration of Nations", European Economic Review, 40 (3-5), p. 697-705.

[3] Bolton, P. and G. Roland, 1997, "The Breakup of Nations: A Political Economy Analysis", Quarterly Journal of Economics, 112 (4), p. 105790.

[4] Culver, S.E. and D.H. Papell, 1997, "Is there a unit root in the inflation rate? Evidence from sequential break and panel data models", Journal of Applied Econometrics, 12(4), p. 435-444.

[5] De Grauwe, P., 2000, Economics of monetary union, 4th. ed. (Oxford University Press, Oxford UK). 
[6] Dixit, A.K., 1992, "Investment and hysteresis", Journal of Economic Perspectives, 6-1, p. 107-132.

[7] Dixit, A.K., 1993, The art of smooth pasting (Fundamentals of pure and applied economics vol. 55) (Harwood Academic Publishers, Amsterdam).

[8] Dixit, A.K. and R.S. Pindyck, 1994, Investment under uncertainty (Princeton University Press, Princeton NJ).

[9] Fidrmuc, J., 1999, "Stochastic Shocks and Incentives for (Dis)Integration", CEPR Discussion Paper no. 2104 (Centre for Economic Policy Research, London).

[10] Gros, D. and N. Thygesen, 1998, European Monetary Integration, 2nd ed. (Longman, Harlow UK).

[11] Lai, K.S., 1997, "On the disparate evidence on trend stationarity in inflation rates: A Reappraisal", Applied Economics Letters, 4(5), p. 305-309.

[12] Pindyck, R.S., 1991, Irreversibility, uncertainty, and investment, Journal of Economic Literature, XXIX, p. 1110-1148.

[13] Strobel, F., 2000, "Monetary integration and the value of waiting", in: Moser, T. and B. Schips (eds.), EMU, financial markets and the world economy (Kluwer Academic Publishers, Boston MA), p. 181-192.

[14] Strobel, F., 2001, "When to leave a monetary union?", Revue Economique, 52(2), p. 389-397.

[15] Strobel, F., 2002, "Leaving a monetary union: expected time and probability", Greek Economic Review, 22(1), p. 17-22. 


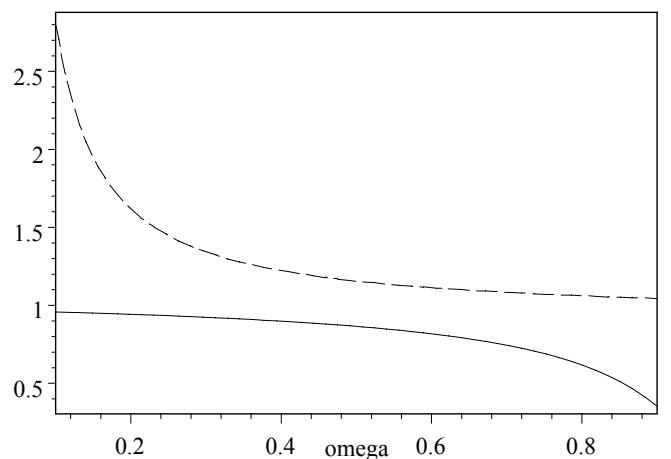

Fig. 1a: $\Upsilon_{a}(-), \frac{1}{\Upsilon_{b}}(--) ; \sigma=0.02, \rho=0, \mu=0.06, \tau=0.25$

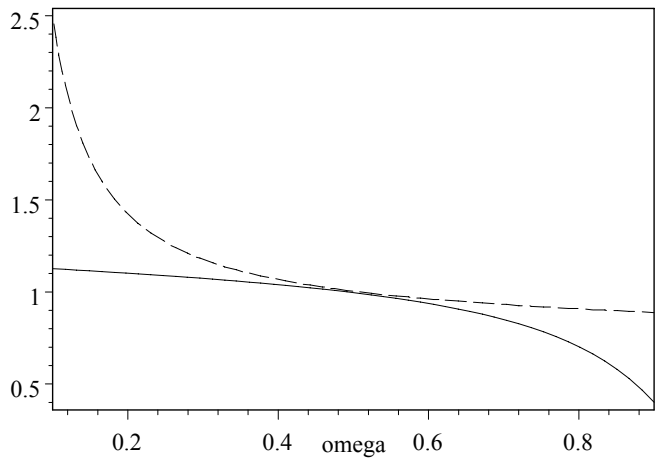

Fig. 1b: $\Upsilon_{a}(-), \frac{1}{\Upsilon_{b}}(--) ; \sigma=0.06, \rho=0, \mu=0.06, \tau=0.25$

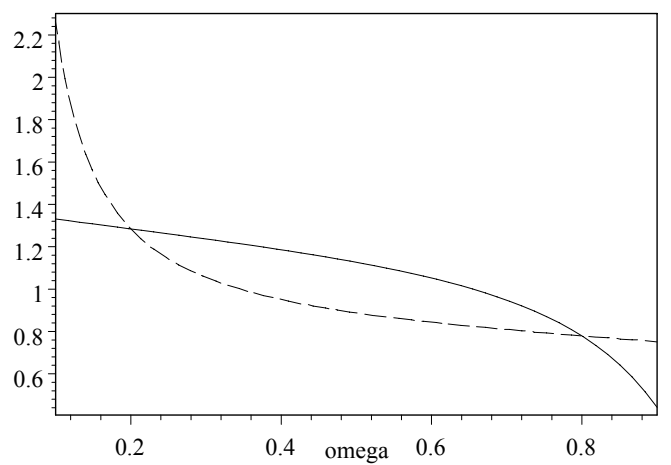

Fig. 1c: $\Upsilon_{a}(-), \frac{1}{\Upsilon_{b}}(--) ; \sigma=0.1, \rho=0, \mu=0.06, \tau=0.25$ 


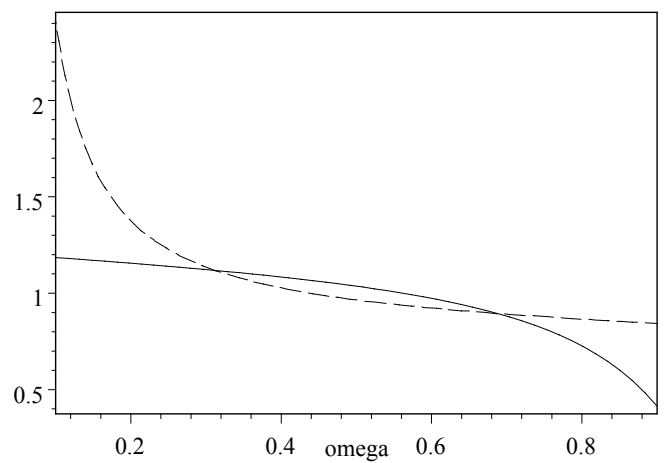

Fig. 2a: $\Upsilon_{a}(-), \frac{1}{\Upsilon_{b}}(--) ; \sigma=0.06, \rho=-0.5, \mu=0.06, \tau=0.25$

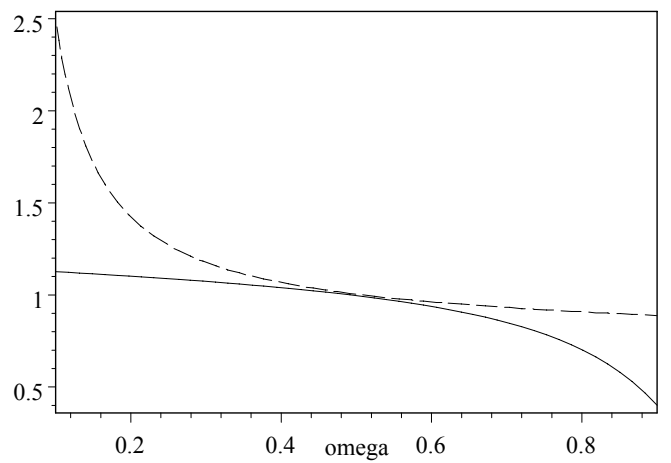

Fig. 2b: $\Upsilon_{a}(-), \frac{1}{\Upsilon_{b}}(--) ; \sigma=0.06, \rho=0, \mu=0.06, \tau=0.25$

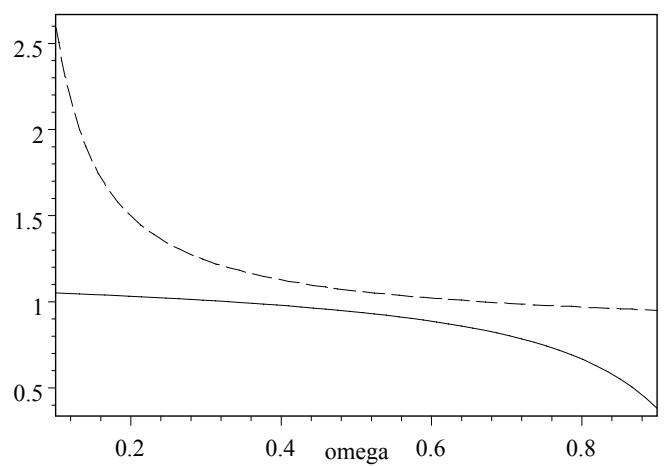

Fig. 2c: $\Upsilon_{a}(-), \frac{1}{\Upsilon_{b}}(--) ; \sigma=0.06, \rho=0.5, \mu=0.06, \tau=0.25$ 


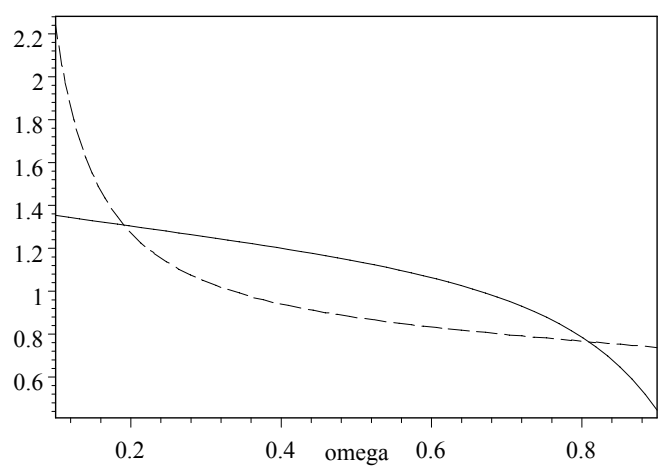

Fig. 3a: $\Upsilon_{a}(-), \frac{1}{\Upsilon_{b}}(--) ; \sigma=0.06, \rho=0, \mu=0.02, \tau=0.25$

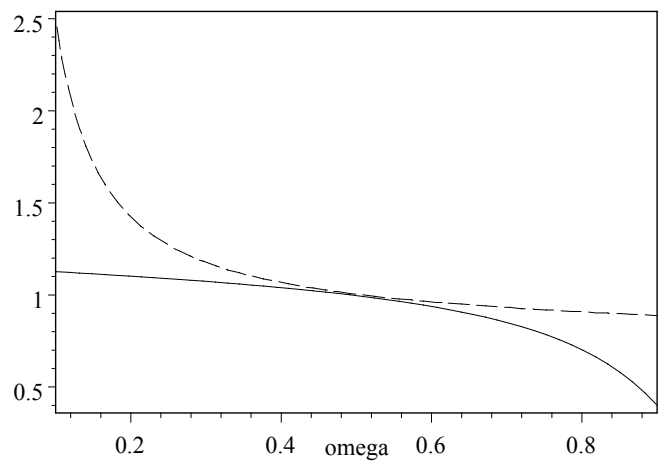

Fig. 3b: $\Upsilon_{a}(-), \frac{1}{\Upsilon_{b}}(--) ; \sigma=0.06, \rho=0, \mu=0.06, \tau=0.25$

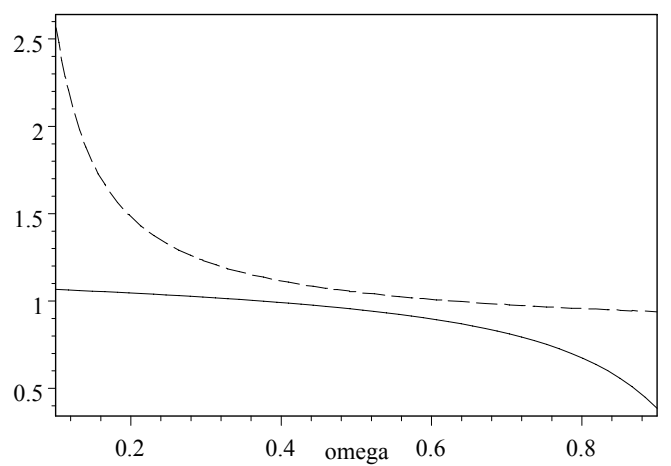

Fig. 3c: $\Upsilon_{a}(-), \frac{1}{\Upsilon_{b}}(--) ; \sigma=0.06, \rho=0, \mu=0.1, \tau=0.25$ 


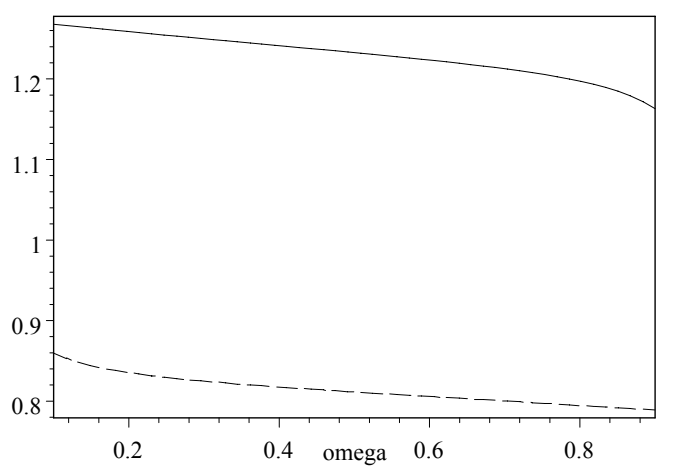

Fig. 4a: $\Upsilon_{a}(-), \frac{1}{\Upsilon_{b}}(--) ; \sigma=0.06, \rho=0, \mu=0.06, \tau=0.01$

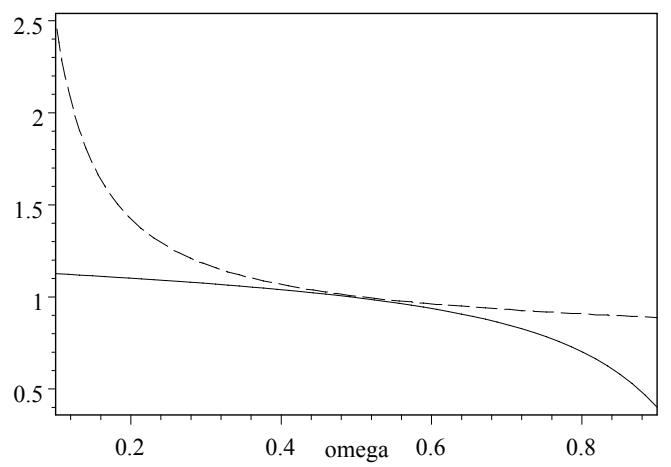

Fig. 4b: $\Upsilon_{a}(-), \frac{1}{\Upsilon_{b}}(--) ; \sigma=0.06, \rho=0, \mu=0.06, \tau=0.25$

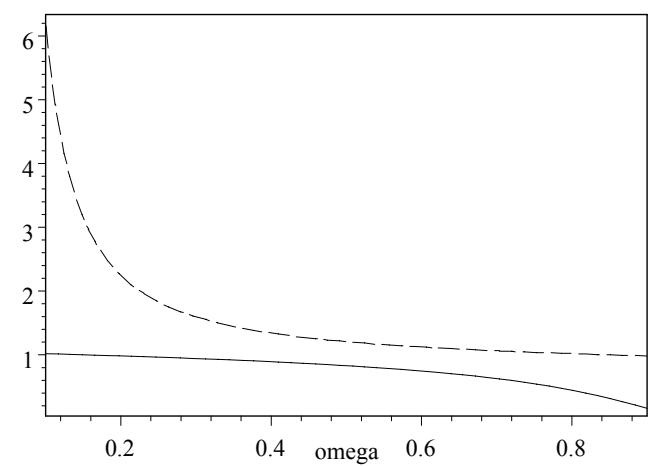

Fig. 4c: $\Upsilon_{a}(-), \frac{1}{\Upsilon_{b}}(--) ; \sigma=0.06, \rho=0, \mu=0.06, \tau=0.5$ 